ORNL/TM-2014/216

\title{
Cognitive Determinants in Patient Decision Making for Total Knee Arthroplasty
}

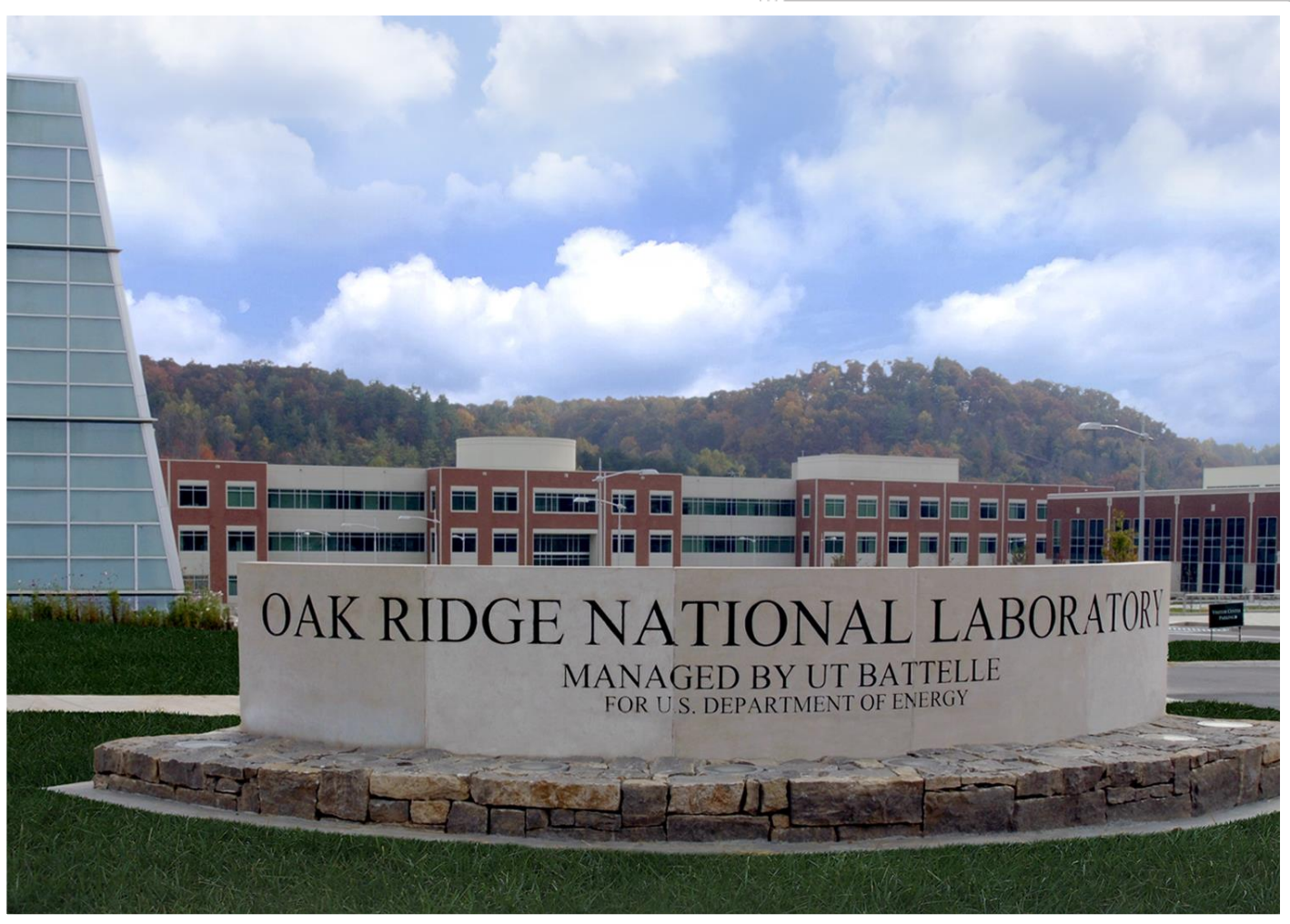

Approved for public release: distribution is unlimited.

Jack C. Schryver 


\title{
DOCUMENT AVAILABILITY
}

Reports produced after January 1, 1996, are generally available free via US Department of Energy (DOE) SciTech Connect.

Website http://www.osti.gov/scitech/

Reports produced before January 1, 1996, may be purchased by members of the public from the following source:

\author{
National Technical Information Service \\ 5285 Port Royal Road \\ Springfield, VA 22161 \\ Telephone 703-605-6000 (1-800-553-6847) \\ TDD 703-487-4639 \\ Fax 703-605-6900 \\ E-mail info@ntis.gov \\ Website http://www.ntis.gov/help/ordermethods.aspx
}

Reports are available to DOE employees, DOE contractors, Energy Technology Data Exchange representatives, and International Nuclear Information System representatives from the following source:

Office of Scientific and Technical Information

PO Box 62

Oak Ridge, TN 37831

Telephone 865-576-8401

Fax 865-576-5728

E-mail reports@osti.gov

Website http://www.osti.gov/contact.html

This report was prepared as an account of work sponsored by an agency of the United States Government. Neither the United States Government nor any agency thereof, nor any of their employees, makes any warranty, express or implied, or assumes any legal liability or responsibility for the accuracy, completeness, or usefulness of any information, apparatus, product, or process disclosed, or represents that its use would not infringe privately owned rights. Reference herein to any specific commercial product, process, or service by trade name, trademark, manufacturer, or otherwise, does not necessarily constitute or imply its endorsement, recommendation, or favoring by the United States Government or any agency thereof. The views and opinions of authors expressed herein do not necessarily state or reflect those of the United States Government or any agency thereof. 
Computational Science \& Engineering

\title{
COGNITIVE DETERMINANTS IN PATIENT DECISION MAKING FOR TOTAL KNEE ARTHROPLASTY
}

\author{
Jack C. Schryver
}

Date Published: September 2014

Prepared by

OAK RIDGE NATIONAL LABORATORY

Oak Ridge, Tennessee 37831-6283

managed by

UT-BATTELLE, LLC

for the

US DEPARTMENT OF ENERGY

under contract DE-AC05-00OR22725 



\section{CONTENTS}

CONTENTS
LIST OF FIGURES
ACKNOWLEDGMENTS
ABSTRACTT
1.
2.
3.





\section{LIST OF FIGURES}

Figure

1. Phases of an episode of care for total knee arthroplasty. .............................................................. 2

2. Simulation user input parameters viewed from the RePast Simphony user panel................................. 9

3. Effect of System 1 weight on proportion of agents having surgery ................................................... 10

4. Effect of information avoidance strength on proportion of patients having surgery........................... 11

5. Mitigating effect of "shopping around" for OS providers on rationing by wait time........................... 12 



\section{ACKNOWLEDGMENTS}

This research was supported by the ORNL Laboratory Director's Research and Development fund. 


\begin{abstract}
We constructed a novel agent-based simulation where patients and providers interact within the context of an episode of care within a healthcare ecosystem. The model examined the influence of three psychosocial factors on individuals experiencing chronic knee pain on their decision to undergo total knee arthroplasty: (1) dual processing decision making modes; (2) information avoidance bias; and (3) rationing of healthcare by wait time. Greater reliance on the fast intuitive System 1, in contrast with the slow deliberative System 2, resulted in greater proportions of knee joint pain sufferers to seek surgical solutions. Increasing bias to avoid unwanted information reduced the proportion of patients seeking surgery. Finally, increased willingness to minimize wait time by "shopping around" for additional OS providers decreased rationing of provider services and greater proportions of patients opted for surgical solutions. The simulation results indicated that health cognition can play a significant role in patient decision making. Future directions for model development are discussed.
\end{abstract}

\title{
1. INTRODUCTION
}

Cognitive factors are significant but often neglected elements that influence patient decision-making during prospective elective episodes of medical care. This paper presents a case study of total knee arthroplasty (TKA) to illustrate the interplay of cognitive factors in an agent-based simulation (ABS) of individual patient behavior during an episode of care beginning prior to and terminating 90 days after undergoing this surgical procedure.

TKA is a surgical procedure performed when a patient's knee no longer functions or causes severe pain. It is a common solution undertaken by many people who suffer from severe osteoarthritis. Conventional treatment of knee pain ranges from non-steroidal anti-inflammatory drugs, narcotic and non-narcotic (weak) analgesics and physical therapy [1]. However, in advanced cases of osteoarthritis these treatments may be of limited value, leaving TKA as the best option to alleviate pain and restore function. However, these potential benefits of surgery also need to be weighed against the risks of surgical mortality and morbidity [2].

An episode of care around TKA, or total knee replacement surgery, is an elective medical procedure that can be decomposed into several distinct phases, as depicted in Fig. 1.

At the most abstract level these phases are: (1) pre-assessment; (2) pre-admission (approximately 30 days); (3) procedural; and (4) post-surgical (approximately 90 days). During pre-assessment, the individual suffers from knee joint pain and loss of function, often due to osteoarthritis or injury. The individual may seek help from a primary care physician (PCP), undergo imaging such as X-ray or MRI, and/or receive care in the form of pain-relieving medication. During the pre-assessment phase, patients consult with an orthopedic surgeon (OS) by referral from a PCP, self-referral or referral from another OS. An initial assessment is performed by the OS that may include diagnostic imaging, physical examination, and elicitation of information from the patient to help in the determination of the necessity of TKA. During this period of assessment, the OS weighs patient expectations or attempts to direct patient expectations toward more realistic goals, and determines whether the individual is a suitable candidate for TKA [3]. Following a positive joint determination by the OS and patient that TKA is indicated, the surgery can be scheduled. Many factors are involved in this shared decision making process as illustrated by the large geographic variation found in Medicare rates for TKA [4]. This stage is followed by hospital pre-admission, which occurs three days before the surgery, and consists of pre-blood work and ECG, consultation and signoff by the PCP or cardiologist. The TKA itself is typically performed as an inpatient procedure in a hospital, but also may be performed at an ambulatory surgical center. The episode of care 


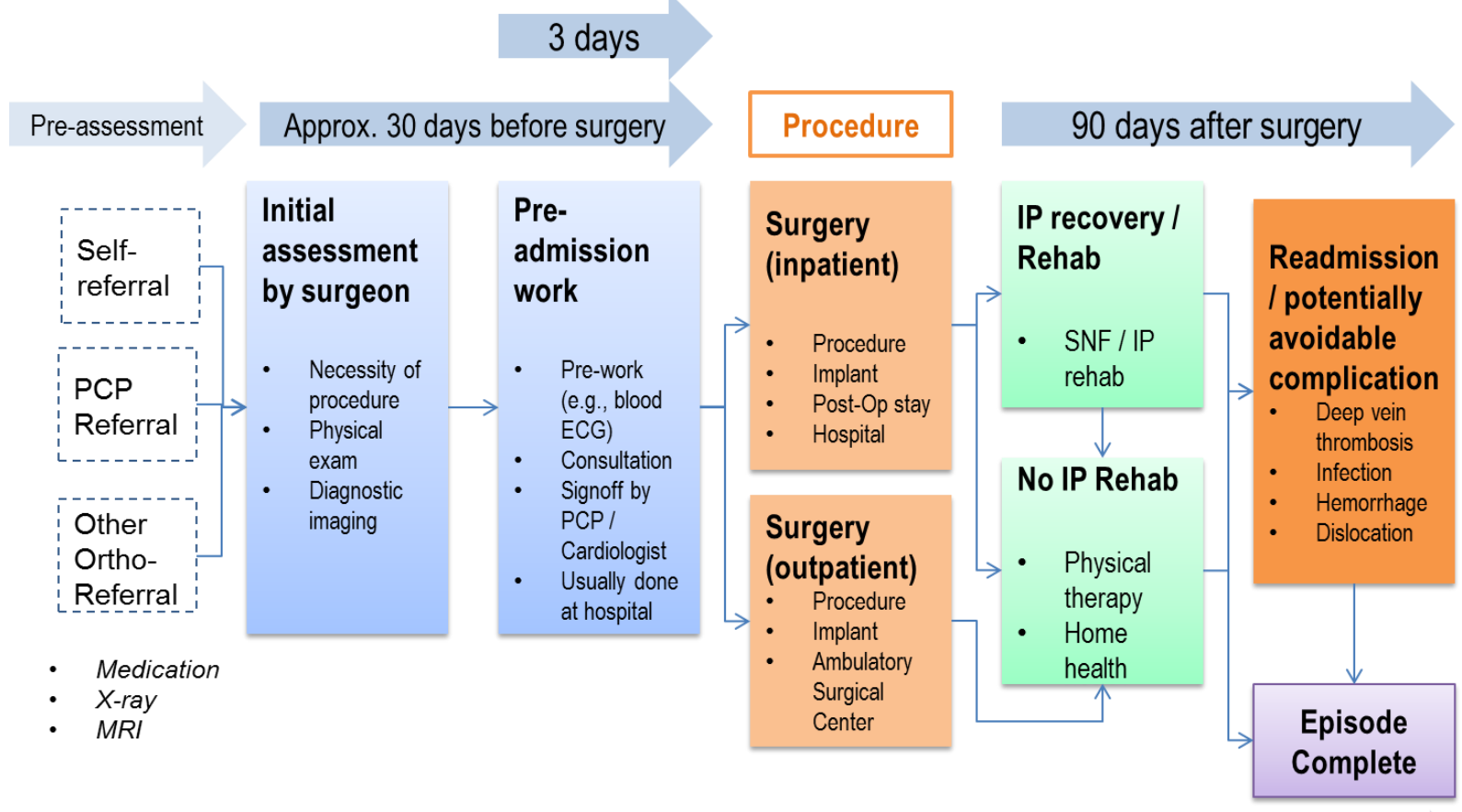

Care Progression Model

Fig. 1. Phases of an episode of care for total knee arthroplasty.

is terminated 90 days after completion of the TKA. During the first stage of this 90 -day period the patient undergoes physical rehabilitation and recovery from the procedure for the purpose of relieving postsurgical pain and recovering knee joint function. The patient may face a wide range of rehabilitation options. In the absence of significant provider attempts at consultation or intervention, the choice of rehabilitation facility is largely defaulted to patient preferences. For example, a patient may prefer more expensive inpatient options such as a hospital or a skilled nursing facility. Other options are physical therapy and home health care. A limited percentage of cases will require hospital readmission prior to the end of the episode due to potentially avoidable complications such as deep vein thrombosis, infection, hemorrhage or dislocation, leading to additional costs directly associated with the episode of care.

\section{HEALTH COGNITION}

Historically, the study of human cognition was compared to a normative benchmark with a foundation in rational choice theory. The classical approach in economics to choice among risky prospects is expected utility theory (EUT) [5]. This theory posits that each potential outcome is paired with a utility that expresses its explicit value on a single dimension. If the probabilities of the outcomes can be estimated, the EU of a choice is the additive combination of the utilities of all potential outcomes weighted by their probabilities of occurrence. The normative approach has been used extensively by the medical community in drafting recommended decision making protocols for clinical judgment.

In contrast, the psychological literature in recent decades has emphasized a more descriptive approach to human decision making that is characterized by bounded rationality, use of heuristics, and cognitive bias. Several investigators have applied this paradigm to patient/provider decision making. For example, the EUT assumption that outcome probabilities that sum to unity can be determined has been challenged by the unpacking principle which states that providing a more detailed description of an outcome increases 
its judged likelihood. Framing and adding decision alternatives can distort preferences for alternatives being considered. Cognitive bias also plays an important role in structuring medical decision problems through sunk costs, loss aversion, regret, discounting, omission, optimism and pessimism [6-8]. Knowing how to apply the principles of health cognition to decision models is crucial in constructing accurate models of behavior.

\section{AGENT-BASED SIMULATION}

The practice of ABS [9] has begun to enter a somewhat more mature phase of development, at least with respect to dynamic analysis of socio-technical systems, although it still has not fully penetrated some knowledge domains as a commonly-accepted approach to simulation, including the analysis of healthcare systems. There are, however, a few published examples of ABS models in the healthcare sector, including models of congestive heart failure [10], type 1 diabetes mellitus patient adoption of continuous glucose monitoring technology [11], healthcare delivery systems for type 2 diabetes [12], and health behaviors such as the spread of obesity [13] and drinking behavior [14].

Despite the limited use of ABS in the healthcare sector to date, there are many advantages to a disaggregated, individual-based approach to complex systems simulation as compared to a macro approach such as systems dynamics simulation. ABS has the capability to mimic the behavior of systems in which the proliferation of complex or heterogeneous individual agent profiles can produce complex patterns of behavior, or there exists extensive interactions among agents. For these reasons we elected to develop a preliminary model of a total knee replacement episode of care in an agent-based simulation framework.

\section{MODEL DESIGN}

An individual-based model was developed in the agent-based simulation package Repast Simphony (Java version). RePast Simphony implements agent protocols with discrete time management for which all actions are synchronized in time by equal-step updating. The initial version of this model was fairly simple, requiring only interactions between patient (ReplacementSurgeryPatient) and OS (OrthoSurgeon) agents to capture the main behavioral phenomena of interest. A simple fee-for-service payment mechanism was assumed to measure outcomes in terms of dollars spent and medical service utilization. The model implements the sequential stages of TKA shown in Figure 1. The pre-assessment stage was not modeled in this initial version of the software.

\subsection{KNEE JOINT PAIN}

The primary stimulus for seeking treatment on the part of the patient is the experienced degree of knee joint pain relative to an individual's pain tolerance threshold $(l)$. The initial amount of knee pain $\left(p_{0}\right)$ and individual pain threshold are set independently for each patient by random draws from uniform distributions. The distributions were chosen such that almost half the patient population will experience

pain exceeding their personal threshold at the start of a simulation run. It is assumed that in the absence of treatment, knee pain increases monotonically with time. Here we assume that a single time step corresponds to one day. During each simulation update, which occurs every time step (day), experienced knee pain is incremented by one with probability $k_{l}=.01$. The patient performs a cognitive comparison at each time step in order to revise their belief that a surgical option is required to mitigate experienced pain. The belief function $\left(B_{p}\right)$ assumes a two-parameter logistic form as shown in Eq. 1. 


$$
B_{p}=1 /\left[1+e^{\theta(c-(n-l))}\right]
$$

The range of the belief function is the interval $(0,1)$. If the free parameter $c=0$ the degree of belief in treatment takes on a value of 0.5 whenever experienced pain $n$ equals the pain tolerance threshold $l$. The scaling parameter $\theta$ establishes the height of the function in the domain of values used in the simulation experiments. Following TKA the knee pain attributable to the chronic condition is reset to zero. Pain resulting from TKA itself is not considered in this episodic model.

After some experimentation we selected parameter values $\mathrm{c}=80$ and $\theta=.01$ for the simulation experiments reported in this paper. It was discovered that the influence of other free parameters in the simulation, especially information avoidance strength, was highly sensitive to the selected parameters for the pain-tobelief conversion function. Simply stated, decreasing the localized slope of the conversion function tended to increase the sensitivity of information avoidance strength to the value of the output variables.

\subsection{TREATMENT SEEKING DECISION MODEL}

In economic theory the dominant framework for decision making models is still EUT. We adopted a descriptive approach to human decision making in order to mimic an alternative decision process implicated in seeking treatment (surgery) to alleviate knee joint pain. One widely-accepted framework, named dual processing theory, posits the existence of two cognitive systems that are responsible for formulating decisions. This theory characterizes decision making as a flexible integration of a fast, intuitive, automated and affective experiential system (System 1) and a slow, analytical, deliberative and rational system (System 2). Mukherjee developed a mathematical model that aggregates System 1 and 2 processes into a single decision rule [15]. He assumed that evaluation of risky choice involved the linear combination of the quantitative outputs of the two systems to generate a single outcome or decision. Dual processing is applicable to the episodic healthcare delivery context because the patient can either attend to the emotional quality generated by the pain stimulus or objectively weigh the risks and benefits of surgery, depending on which imperative is salient at any given moment. The Mukherjee model was adapted to medical decision making in [16]; Eq. 2 shows the value function $V$ for a single choice alternative $(C)$ in that adaptation,

$$
V(C)=\gamma \frac{1}{n} \sum_{i} x_{i}^{m_{I}}+(1-\gamma) w \sum_{i} p_{i} x_{i}
$$

where $p_{i}$ is the probability of outcome $x_{i}, 0 \leq m_{I} \leq 1$, and $0 \leq \gamma<1$ is the relative weight given to System 1 on the RHS. The value functions in Eq. 2 for System 1 (left-hand term on RHS) and System 2 (right-hand term on RHS) have distinctively different forms. System 1 value is expressed primarily as a power value function. With a fractional exponent the System 1 value function satisfies risk aversion for gains because the function is concave when the utility $\left(x_{i}\right)$ is greater than zero. We set $m_{l}=0.5$ for all simulations reported in this paper. Note that outcome probabilities are absent from the System 1 value function, i.e., every outcome is equally weighted. This highlights an important feature of the intuitive System 1 mode, which can be described as a type of satisficing that enumerates potential outcomes, especially affectivelytinged outcomes, without regard to the likelihoods of their occurrence. The salience of potential outcomes is what counts, not their probability of occurrence. The right-hand term of Eq. 2 signifies the classical utility function, where values $\left(x_{i}\right)$ are preserved veridically in subjective calculations, and utilities are properly weighted by their probabilities $\left(p_{i}\right)$. Finally, $w$ is a scaling constant which can be set to unity.

Djulbegovic et al. [16] consider binary choice where, for example, a patient either opts for treatment (surgery) or elects to avoid/postpone treatment. Since according to some objective criteria (e.g., clinical guidelines) surgery is either indicated or not indicated regardless of patient preferences, we can identify four different outcomes and their associated utilities (Table 1). 
Table 1. Decision outcome matrix (values)

\begin{tabular}{|l|cc|} 
& Seek surgery & Don't seek surgery \\
\hline Surgery indicated & $\mathrm{X}_{1}=180$ & $\mathrm{X}_{3}=30$ \\
\hline Surgery not indicated & $\mathrm{X}_{2}=10$ & $\mathrm{X}_{4}=70$ \\
\hline
\end{tabular}

The critical informational quantities processed by the patient are net benefits and net harms. The net benefit of treatment is equal to the difference in outcomes of seeking and not seeking surgery when medical guidelines indicate surgery. The net benefit attributed by System 1 is denoted by $B_{1}$, and System 2 net benefits are denoted by $B_{2}$. Similarly, the difference in outcomes of seeking and not seeking surgery when it is not indicated is defined as the net harms. Net harms are accordingly labelled $H_{l}$ for System 1 and $\mathrm{H}_{2}$ for System 2. Net benefits/net harms are assessed differently according to System 1 and System 2 modes of thinking. We can use net benefits/net harms to compute the threshold probability $\left(p_{t}\right)$, where surgery is actually indicated with respect to unbiased medical guidelines, but at which the patient is indifferent with respect to deciding between surgery and avoidance (Eq. 3).

$$
p_{t}=\left(\frac{1}{1+\frac{B_{2}}{H_{2}}}\right)\left[1+\frac{\gamma}{2(1-\gamma)}\left(\frac{H_{1}}{H_{2}}\right)\left(1-\frac{B_{1}}{H_{1}}\right)\right]
$$

The indifference threshold $\left(p_{t}\right)$ is interpreted as follows. If the probability (or belief) that surgery is indicated $\left(B_{p}\right)$ exceeds $\mathrm{p}_{\mathrm{t}}$ the patient prefers the surgical option, else some option other than surgery is adopted. The first term in parentheses to the left of the square-bracketed phrase refers to calculations used in EUT, which depends only on the System 2 net benefits/net harms ratio. Here, increasing the ratio lowers the indifference threshold. On the other hand, if an examination of System 1 net harms and net benefits reveals $H_{1}>B_{1}$, the indifference threshold is higher than the EUT threshold. In this case, $H_{1}>H_{2}$ further elevates the indifference threshold, but if $H_{l}<H_{2}$ the opposite trend holds.

\subsection{INFORMATION AVOIDANCE}

Research into communication and cognition has long characterized human beings as curious, informationseeking organisms. Likewise, a dominant trend in health informatics emphasizes patient empowerment and the health benefits it accrues through increased access to health information and greater shares in treatment decision making; nevertheless, in certain instances people prefer to remain uniformed and information may be selectively filtered out through intervention of a cognitive bias known as information avoidance [17].

It seems obvious that people may selectively avoid information out of fear of cognitive, affective and behavior consequences of hearing the information. For example, it has been suggested that individuals avoid information if they perceive that it threatens their need for autonomy. A physician tells a patient that someone at high risk for diabetes should give up fried foods, thus motivating a threat response on the part of the patient because she is being asked to cede autonomy with respect to dietary preferences [18]. Blocking the information is therefore viewed as a coping strategy intended to reassert patient autonomy and preserve the status quo. There exists some experimental evidence that health information is avoided in realistic scenarios when feedback may obligate the patient to undesirable behaviors. In one study, participants completed an online risk calculator for a fictitious condition, and were given the opportunity to receive feedback from the calculator that would obligate them to a cervical exam and medication for 
the rest of their life if they were calculated to be at high risk. These participants declined to receive the risk feedback significantly more often than others who were told that the feedback might lead to only slightly undesirable consequences (having their cheek swabbed and taking medication for two weeks.) [18]. Other work discusses avoidance of information derived from genetic screening for cancer [19].

The presence of this kind of selective inattention or avoidance in decision-making most likely implicates System 1 functioning. An important side-effect of System 1 function is the anticipated regret that occurs when a patient realizes the expected "harm" that would occur when an OS provides unwanted information. The discomfort associated with TKA is certainly the type of news that may be received as unwanted. If the individual is physically active, it could also imply a significant loss of autonomy in the sphere of physically demanding activities such as sports, exercise, travel, yard work, etc. Therefore, we expect that a certain proportion of patients who find themselves in the pre-assessment stage will actively avoid information that moves them closer toward arthroplastic solutions to their experienced knee pain.

Although information avoidance can be considered a response triggered by specific situations, some researchers have postulated that there is a generalized avoidance trait leading to either an "open" or "closed" mind. Information-seeking styles are then characterized as either "monitoring", or active scanning of the environment for threats, or "blunting", i.e., avoidance or distraction from threatening information [19]. In this episodic model, each patient is biased toward information-seeking style of avoidance according to a user-selected probability. If the information avoidance bias trait is tagged to a particular patient, its strength is constant over the entire population of patients, and the population parameter value for strength $(0<k \leq 1)$ is set by the user. Otherwise the patient is treated as a "monitor". We assume that the attachment of information avoidance bias to patient agents decrements the calculated net benefits for the case where surgery is indicated by "blunting" the value of the information. This effect is implemented in the episodic model by multiplying the net benefits from System $1\left(B_{l}\right)$ by $(1-k)$, where $k$ is the avoidance parameter, and greater values of $k$ diminish the utility of the net benefit.

\subsection{RATIONING BY WAIT TIME}

Health care rationing is a controversial and sensitive topic in the U. S., although forms of it have been adopted as public policy in some countries. One proven rationing strategy to reduce total healthcare costs, without formulating an explicit rationing policy, is to effectively ration care by increasing the wait time for certain elective surgery. Wait time is a characteristic of queues, in this case, queues formed by patients of specific OS providers scheduled for TKA. If a specific OS always performs an arthroscopic surgery in a constant amount of time with very little variability, wait time is approximately a linear function of queue length. Wait lists are effective rationing devices because although patient time is not literally wasted in a physical queue, the utility of the surgery as an economic good or service declines as a function of time because of discounting (i.e., the value of a good declines as the time required to accrue its benefits increases.) This is the situation that obtains for people waiting for elective surgery in the United Kingdom National Health Service [20].

We model the patient/OS agent population as a simple queuing eco-system with finite numbers of patients and OS providers. Each OS provider has its own surgical queue or waiting list, imposing a resource limitation on the performance of the healthcare system if the patient/provider ratio is high enough relative to provider throughput. Patients exit the eco-system (and the OS queue) following rehabilitation/readmission when the health episode terminates. Patients entering the system at the initial assessment phase shop for a provider with an individually-acceptable wait time. This is a simplification of the theory that only patients with a high valuation of time will shop for providers, e.g. [21], by assuming that all patients possess a strict threshold tolerance for waiting. We limit patient search by assuming that every patient contacts exactly $n_{o s}$ OS providers before making a treatment decision. If the patient opts for TKA to relieve their knee pain, they randomly query the queue lengths and throughput of $n_{o s}$ providers. If 
at least one of the OS providers has an estimated wait time (calculated by multiplying the constant provider throughput by queue length) that does not exceed the patient's maximum wait time ( $k_{3}=20$ days), they always schedule TKA with the OS provider possessing the lowest estimated wait time.

\section{MODEL IMPLEMENTATION}

We populated an artificial healthcare ecosystem with patients experiencing knee pain (thousands to tens of thousands) and OS providers (thousands) in RePast Simphony Version 1.0 (Java). Each OS provider was assigned a random surgical throughput value from a uniform distribution. All patient agents were assigned an experienced level of pain from a uniform distribution in the interval $[0,100]$, and a pain threshold from a uniform distribution in [60,120]. Patient agents were also given a maximum wait time (20 days). After examining the queue length and surgical throughput for a surgeon, the agent compared the earliest possible surgery data to this maximum wait time in order to decide whether or not to schedule a TKA procedure with this surgeon.

The decision model selected for patient agents seeking surgery depends on the assigned utilities for each of the four possible outcomes. Referring back to Table 1, every utility was modeled as a non-negative number. Disutilities (negative utilities) were not used, so that even a bad outcome is assigned a positive (albeit small) utility value. The system 2 net benefit was $180-30=150$; conversely, System 2 net harm was $70-10=60$. The facility choice (made by the patient) for TKA was a hospital with probability $k_{2}=0.75$, and Ambulatory Surgical Center (ASC) with probability $1-k_{2}=0.25$.

The decision process underlying selection of a rehabilitation facility was not modeled in much detail for this initial version of the simulation, although in principle any policy affecting this selection might have a substantial impact on mean total episode cost. Instead, rehabilitation choice was modeled as a random draw from a fixed multinomial distribution, where the probabilities associated with different rehabilitation options were differentiated by two factors: living arrangement and ambulatory status of the patient. Discussions with a local OS provider group ${ }^{*}$ generated the hypothesis that patient living status and ambulatory condition affect patient preferences for post-surgical rehabilitation. Specifically, nonambulatory patients are assumed to have greater preference for inpatient rehabilitation as compared with ambulatory patients. We also expected that patients living alone would prefer inpatient care more than those patients living with another person. We estimated the exact probability values for the simulation based on assumed orderings of the different options. We hypothesized that patients who are not ambulatory prefer a SNF to home-based options, and that among the entire population, options providing more assistance and services to patients are preferred to more self-reliant options. Table 2 shows the probabilities used for the initial set of simulation runs.

Table 2. Probabilities for rehabilitation options by living arrangement and ambulation

\begin{tabular}{|cllllll|}
$\begin{array}{c}\text { Living } \\
\text { arrangement }\end{array}$ & Condition & SNF & $\begin{array}{c}\text { Home } \\
\text { health }\end{array}$ & $\begin{array}{c}\text { Physical } \\
\text { therapy }\end{array}$ & Self & None \\
\hline Living alone & Ambulatory & 0.3 & 0.2 & 0.25 & 0.15 & 0.1 \\
\cline { 2 - 7 } & $\begin{array}{c}\text { NOT } \\
\text { Ambulatory }\end{array}$ & 0.5 & 0.15 & 0.1 & 0.1 & 0.15 \\
\hline $\begin{array}{c}\text { NOT living } \\
\text { alone }\end{array}$ & Ambulatory & 0.2 & 0.25 & 0.3 & 0.2 & 0.05 \\
\cline { 2 - 7 } & $\begin{array}{c}\text { NOT } \\
\text { Ambulatory }\end{array}$ & 0.4 & 0.25 & 0.15 & 0.1 & 0.1 \\
\hline
\end{tabular}

${ }^{*}$ OrthoTennessee, Knoxville TN 
Although we are not aware of any data showing a direct effect of living arrangement on rehabilitation preferences, one study found that patients living alone were older, had greater comorbidity and reported more pain both before and after joint replacement surgery in comparison to patients living with another person [22], which, at least, indicates that living arrangement is an important factor in surgical outcomes.

The risk of potentially-avoidable complications (PAC) arising during the post-surgical phase were assumed to be independent of other factors and outcomes in the simulation. The stationary PAC probabilities were fixed at 0.05/day respectively for deep vein thrombosis, infection, hemorrhage or dislocation.

Costs for services used in the initial simulation runs are given in Table 3. These are approximate costs derived from examination of the clinical literature.

Table 3. Costs for services by category

\begin{tabular}{|lr|}
\hline Service & Cost in dollars \\
\hline Surgery Costs & \\
\hline Hospital TKA & 60000 \\
\hline ASC TKA & 30000 \\
\hline Rehabilitation Costs & 20000 \\
\hline SNF & 5000 \\
\hline Home health & 6000 \\
\hline Physical therapy & 0 \\
\hline Self & 0 \\
\hline None & 25000 \\
\hline Potentially Avoidable Complication Costs & 5000 \\
\hline Deep vein thrombosis & 15000 \\
\hline Hospitalization from infection & 25000 \\
\hline Dislocation & 50000 \\
\hline Revision
\end{tabular}

Several parameters are set by the user in the RePast graphical user interface panel (Fig. 2) prior to each set of simulation runs. Here the probability of agent avoidance for unwanted information is currently set at 0.25 . If present, the bias is enforced at full strength (1.0). The number of OS providers that a patient "shops around" for to minimize their wait time is set at three for all agents in the simulation. The balance between System 1 and System 2 is defaulted at 0.5. Finally, the total numbers of patients and providers is specified in the user panel. 


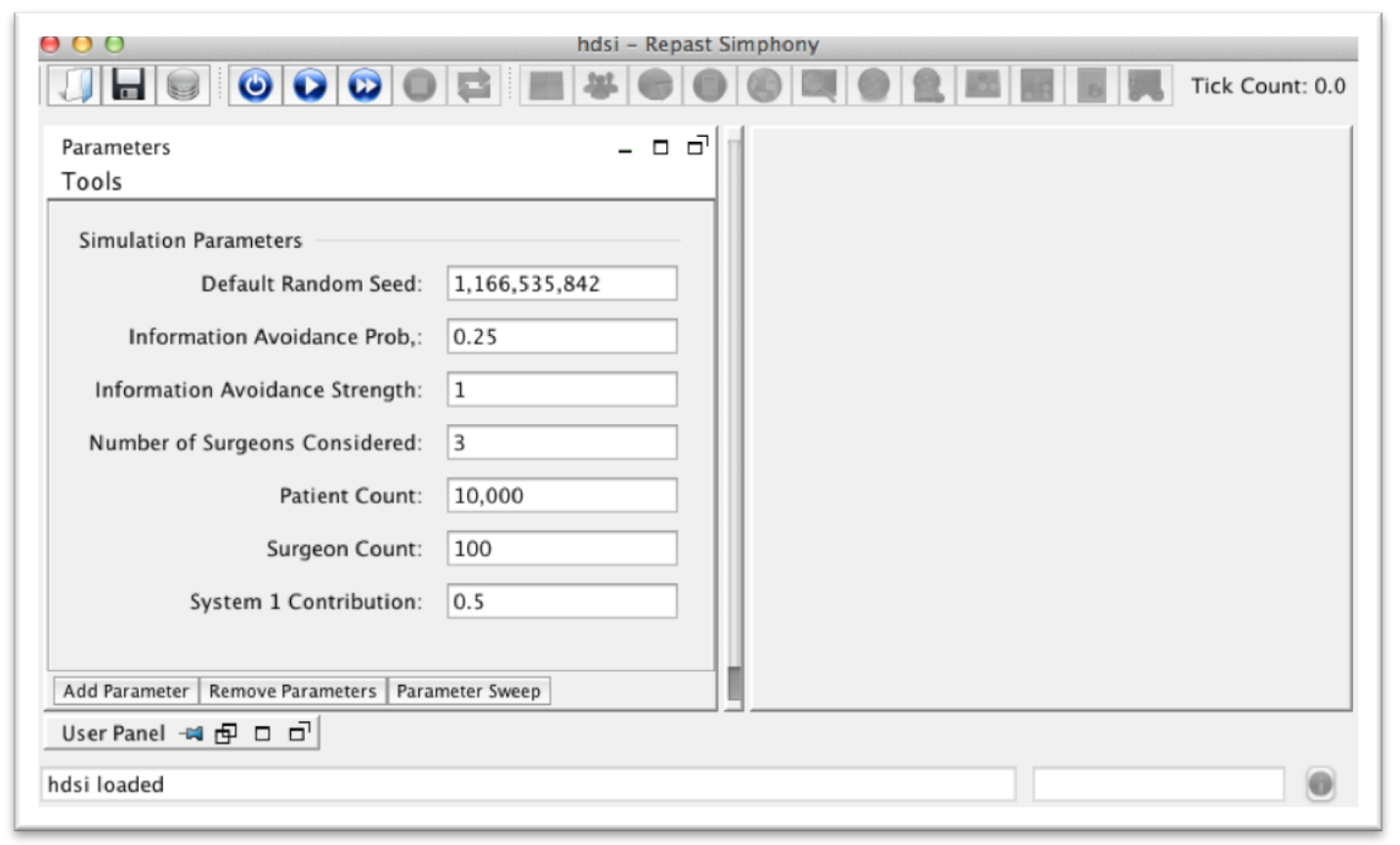

Fig. 2. Simulation user input parameters viewed from the RePast Simphony user panel.

\section{RESULTS OF SIMULATION EXPERIMENTS}

The first simulation experiment established a population of 10000 patients and 1000 OS providers to evaluate the extent of System 1 contribution to treatment decision making over a period of 100 days. A large number of providers was selected because we did not want to overburden the healthcare eco-system for this experiment. Information avoidance was not operational, and only a single OS provider was contacted by each agent. Fig. 3 shows the effect of varying the System 1 contribution parameter $\Upsilon$, by systematically drawing values from the set $\{0,0.25,0.5,0.75,0.99\}$.

The qualitative effects of System 1 weight on surgical proportion and total cost (not shown) were virtually identical. Surgical proportion was a monotonic increasing function of the extent of System 1 influence. The slope of the curve is relatively constant up to $75 \%$ contribution from System 1, but dramatically increases as the System 1 contribution approached unity (99\%). This is the expected result from Eq. 3, which confirms that the main effect of $\Upsilon$ on surgical proportion was unaffected by other factors in the simulation. We cannot conclude from this result that System 1 induces more people to undergo surgery than actually benefit from it (although that is possible), only that it uses a lower threshold to decide in favor of surgery.

The strength of information avoidance bias was assessed in a second simulation experiment using 10000 patient agents and 1000 OS providers. The run length was fixed at 100 time steps (days). Each agent consulted only one surgeon, and preferred an even balance (0.5) between System 1 and System 2. The values of information avoidance bias were drawn from the set $\{0,0.25,0.5,0.75,1\}$. The results of the simulation run are displayed in Fig. 4. 


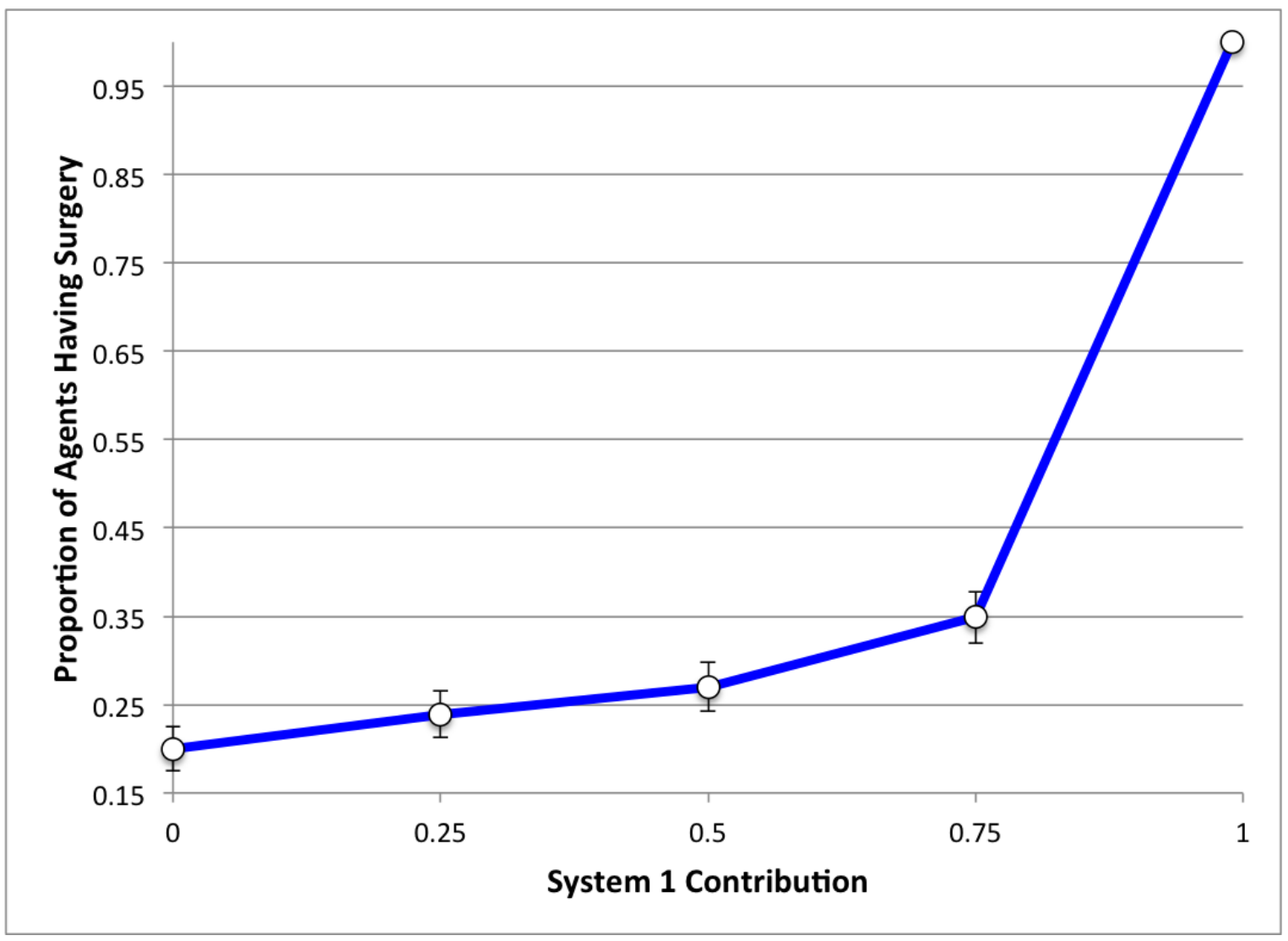

Fig. 3. Effect of System 1 weight on proportion of agents having surgery. Error bars are Wald $95 \%$ confidence intervals where vertical heights (in each direction) are $\mathrm{Z}_{.975}{ }^{*} \mathrm{sqrt}(\mathrm{p}(1-\mathrm{p}) / \mathrm{n})$ and $p$ refers to proportion of agents having surgery.

Fig. 4 confirms the expectation that increasing bias against unwanted information reduces the proportion of patients electing to have surgery. There appears to be a soft floor effect beginning at an information avoidance strength $=0.5$, when the negative slope of the function nearly flattens. Again, the main effect of information avoidance strength appears to not have been distorted by interactions with other model parameters.

The third simulation experiment systematically varied the number of OS providers contacted by 10000 patients before making a decision about arthroplasty in order to assess the effect on rationing by wait time. The simulation run was set at 100 days, and each agent expressed equal preference for System 1 (0.5) and System 2 (0.5). Information avoidance was not a factor. For this experiment the pain tolerance threshold was shifted downward 20 pain points from a uniform distribution over $(60,120)$ to $(40,100)$. This change meant that an expected $60 \%$ of the patient agents experienced knee joint pain at or above threshold at the beginning of the simulation run. Fig. 5 shows that the total number of surgeries increased as patients widened their fields of OS provider contacts. This general result is demonstrated for two surgeon populations: 100 and 1000; however, the mitigating effect of "shopping around" on rationing was more apparent in the population of 1000 surgeons. This is an expected result, because the probability of finding a surgeon whose schedule meets the threshold cannot decrease with the number of surgeons contacted. The curve for 100 surgeons is nearly flat, indicating that system capacity is reached when using a 100 to 1 patient/provider ratio. When only a single surgeon was contacted, surgery rates were nearly identical for 100 and 1000 surgeons, providing further evidence that "shopping around" behavior is necessary to mitigate the burdensome effects of rationing. 


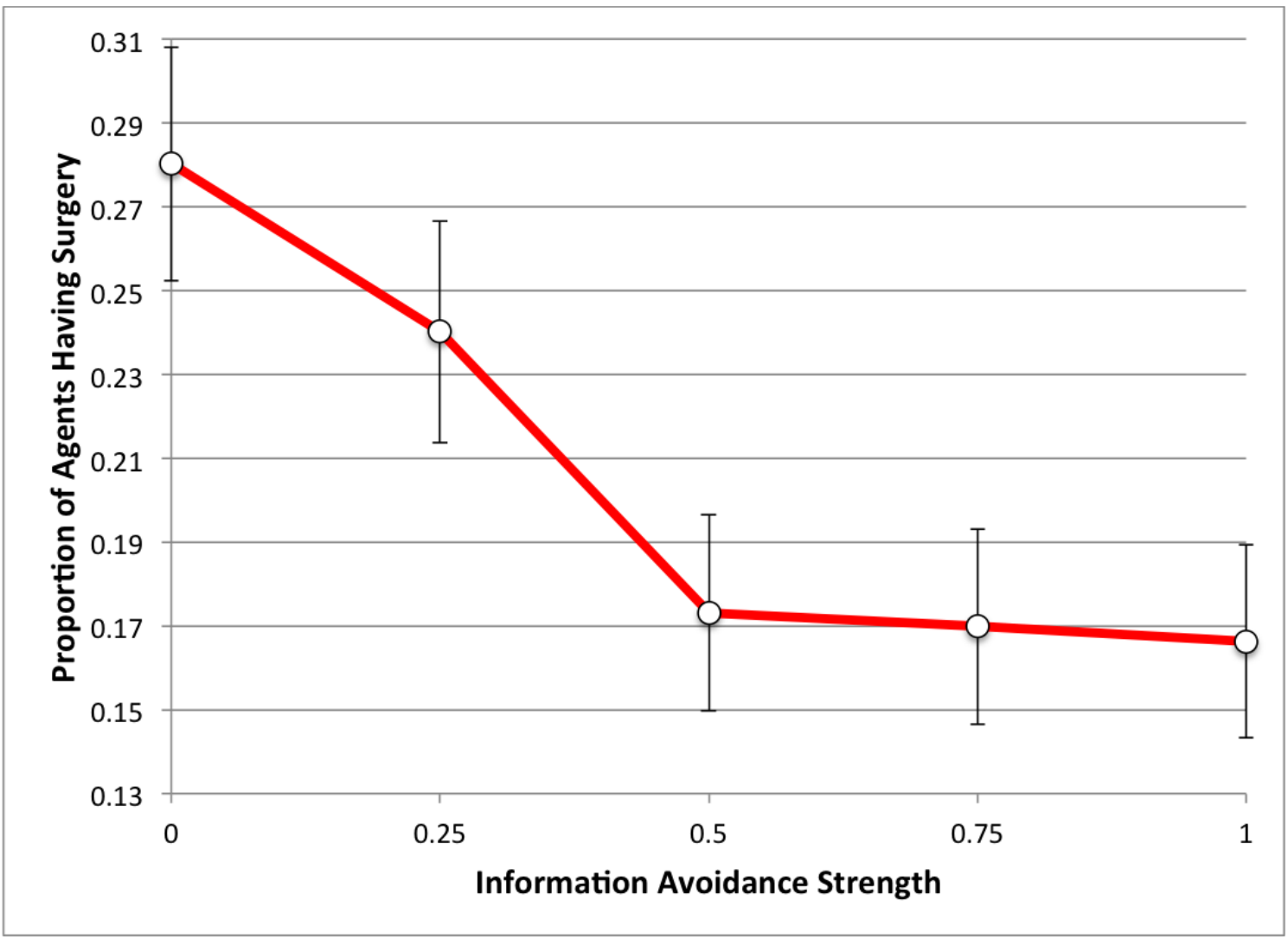

Fig. 4. Effect of information avoidance strength on proportion of patients having surgery. Error bars are Wald $95 \%$ confidence intervals where vertical heights (in each direction) are $Z_{.975} * \operatorname{sqrt}(\mathrm{p}(1-\mathrm{p}) / \mathrm{n})$ and $p$ refers to proportion of agents having surgery. 


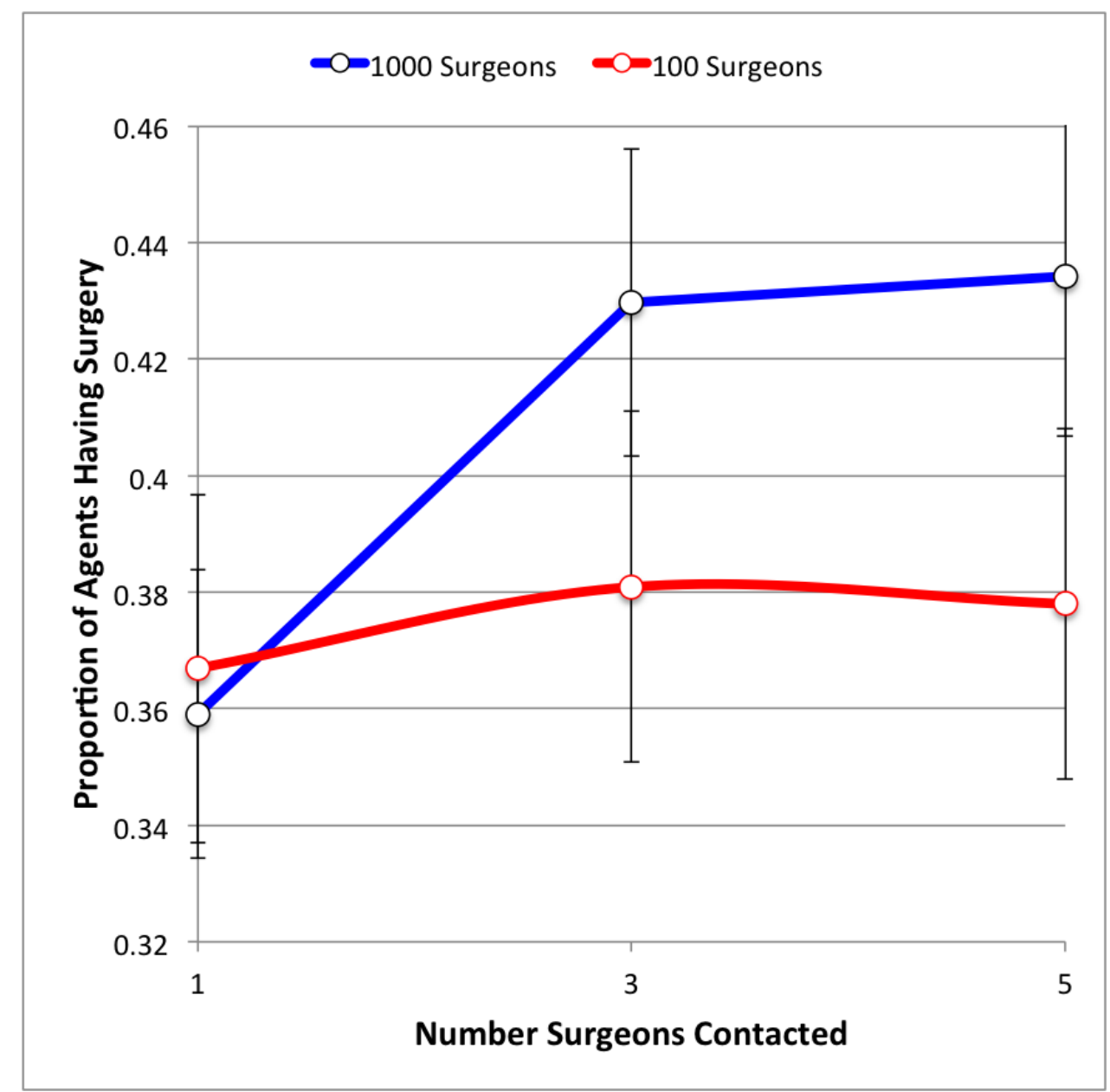

Fig. 5. Mitigating effect of "shopping around" for OS providers on rationing by wait time. Error bars are Wald $95 \%$ confidence intervals where vertical heights (in each direction) are $\mathrm{Z}_{.975} * \operatorname{sqrt}(\mathrm{p}(1-\mathrm{p}) / \mathrm{n})$ and $p$ refers to proportion of agents having surgery.

\section{FUTURE WORK}

The initial version of the simulation model demonstrates the effects of wait rationing, decision mode and information avoidance on a simple episodic care queuing eco-system. The following subsections describe four model upgrades that will extend the ability of the simulation model to address questions regarding the behavior of this system.

\subsection{PRE-ASSESSMENT PHASE AND CONTACT WITH PRIMARY CARE PROVIDER}

A high priority for future development is to create a more detailed descriptive model of the first phase of the TKA process. Initial contact with a primary care provider and subsequent efforts to manage experienced pain and restore function to a disabled knee joint are processes that should be included in the model. The degree of success or failure of pain management at this stage will help to determine choices made later in the process.

Several factors can affect the probability that non-surgical treatments in the pre-assessment phase will be ineffective, and thus propel the patient to the initial assessment phase for surgery. Non-compliance with 
physiotherapy in patients with osteoarthritis of the knee is common. The most important patient-centered factors in non-compliance are "a person's perception of their symptoms, the effectiveness of the intervention, their ability to incorporate it into everyday life and support from physiotherapists." [23].

\subsection{PATIENT DECISION TO SEEK SURGERY TO MITIGATE PAIN}

The decision to consult an OS provider is probably not simply the outcome of individual choice or referral from a primary care provider. Individuals are influenced by attitudes and behaviors of family, friends, and peers. Attitudes about TKA and perceived social norms concerning TKA can also be viewed as products of social learning that occurs through private conversations, mass media messaging and diffusion of content through social media. For example, an individual experience knee joint pain may decide to seek surgical treatment after hearing about a friend's positive experience with TKA. Similar diffusions of health behaviors are documented in the health psychology literature [13, 24]. Locating patients within social networks is the first step in laying the groundwork for these types of diffusions.

\subsection{ORTHOPEDIC SURGEON RECOMMENDATION}

In the current version of the episodic model, the decision to proceed with TKA is largely patient-centered. The factors ostensibly used by the OS to judge the appropriateness of TKA for a specific patient are not considered here, but could, nonetheless, be modeled in some detail. For example, demographic factors (e.g., BMI) and the degree to which patient outcome expectations are reality-based are two factors believed to enter into the calculus underlying an OS recommendation of surgery as a treatment option. The OS provider may also attempt to manage patient expectations directly through intervention at this stage.

The calculus used by the OS provider can be manipulated by imposition of an explicit payment model. For example, a bundled payment model for which the surgeon quarterbacks all medical expenses surrounding an episode of care might incentivize the provider to reduce exposure to costs associated with high-risk patients, such as potentially avoidable complications.

\subsection{PATIENT DECISION ON COURSE OF REHABILITATION}

The path taken toward rehabilitation after TKA has a significant effect on the total cost of elective surgery. Modeling this decision as a patient-driven discrete choice with independent fixed probabilities may be a reasonable approximation to present-day realities. However, viewing this as a shared decision process (or intervention), where the provider explains potential benefits of lower-cost options that shift some of the responsibility onto the patient, may allow the model to calculate the cost benefits of this kind of intervention. For example, motivated patients may derive just as much benefit from receiving physical therapy on an outpatient basis as an extended post-operative stay in a skilled nursing facility.

The realism of the independence assumption can also be questioned. Rehabilitation choice, prior pain management choices, and the hospital/ASC choice of venue for surgery are quite likely decisions governed by a suite of correlated factors. Some of these factors are probably measurable in some explicit sense, e.g., cost, but we might also hypothesize that these healthcare decisions are primarily related to unobservable patient traits such as pre-TKA expectations, self-efficacy, or locus of control.

\section{SUMMARY AND CONCLUSION}

Modeling an episode of healthcare is a natural application of agent-based simulation where patients and providers interact in a healthcare ecosystem. We created an episode of care built around arthroplasty, or 
total knee replacement surgery, in order to study the influence of three psychosocial factors on pain management decisions in greater detail: (1) dual processing modes in decision making; (2) information avoidance bias; and (3) rationing of healthcare by wait time. Allocating greater decision making resources to the fast intuitive System 1, in contrast with the slow deliberative System 2, resulted in greater proportions of patients suffering from knee joint pain to seek surgical solutions. The mean total cost of episodes of care also increased with more emphasis on System 1 based decision styles. Increasing bias to avoid unwanted information reduced the proportion of patients seeking surgical options. Finally, increased willingness to minimize wait time by "shopping around" for additional OS providers decreased rationing and greater proportions of patients were afforded surgical solutions.

Future model development should be guided by the following considerations. The pre-assessment stage of an episode of care should be modeled in greater detail to include pre-surgical approaches to management of knee joint pain. The patient choice to seek treatment, OS provider assessment, and choice of rehabilitation facility are specific decision points along an episode of care that can be explicated through more descriptive models of decision making, leading to better cost estimates by accounting for the effects of cognitive factors that mediate decisions at critical junctures during an episode of care. 


\section{REFERENCES}

1. Adatia, A., K. Rainsford, and W.F. Kean, Osteoarthritis of the knee and hip. Part I: aetiology and pathogenesis as a basis for pharmacotherapy. Journal of Pharmacy and Pharmacology, 2012. 64(5): 617-625.

2. Hamel, M.B., Toth, M., Legedza, A. and M.P. Rosen, Joint replacement surgery in elderly patients with severe osteoarthritis of the hip or knee: decision making, postoperative recovery, and clinical outcomes. Archives of Internal Medicine, 2008. 168(13): 1430.

3. Mancuso, C.A., Graziano, S., Briskie, L.M., Pellicci, P.M., Salvati, E.A. and Sculco, T.P., Randomized trials to modify patients' preoperative expectations of hip and knee arthroplasties. Clinical Orthopaedics and Related Research, 2008. 466(2): 424-431.

4. Weinstein, J.N., Bronner, K.K., Morgan, T.S. and Wennberg, J.E., Trends and geographic variations in major surgery for degenerative diseases of the hip, knee, and spine. Health Affairs, 2004; Supplement Variation: VAR81-89.

5. Schoemaker, P.J., The expected utility model: Its variants, purposes, evidence and limitations. Journal of Economic Literature, 1982: 529-563.

6. Arkes, H., The psychology of patient decision making. Symptom Research: Methods and Opportunities, 2003.

7. Broadstock, M. and S. Michie, Processes of patient decision making: theoretical and methodological issues. Psychology and Health, 2000. 15(2): 191-204.

8. Chapman, G.B. and A.S. Elstein, Cognitive processes and biases in medical decision making. Decision making in health care: Theory, psychology, and applications, 2000: 183-210.

9. Macal, C.M. and M.J. North. Tutorial on agent-based modeling and simulation. in Proceedings of the 37th conference on Winter simulation. 2005. Winter Simulation Conference.

10. Liu, P., An Agent-Based Model to Study Accountable Care Organizations, in Industrial and Systems Engineering2013, University of Southern California.

11. Verella, J.T. and S.D. Patek, Toward an Agent-Based Patient-Physician Model for the Adoption of Continuous Glucose Monitoring Technology. Journal of Diabetes Science and Technology, 2009. 3(2): 353 .

12. Paranjape, R. and S. Gill. Agent-based simulation of healthcare for type II diabetes. in Advances in System Simulation (SIMUL), 2010 Second International Conference on. 2010. IEEE.

13. Bourisly, A.K., An Obesity Agent Based Model: A New Decision Support System for the Obesity Epidemic, in AsiaSim 2013. 2013, Springer. p. 37-48.

14. Gorman, D.M., Mezic, J., Mezic, I. and Gruenewald, P.J., Agent-based modeling of drinking behavior: a preliminary model and potential applications to theory and practice. American Journal of Public Health, 2006. 96(11): 2055-2060.

15. Mukherjee, K., A dual system model of preferences under risk. Psychological Review, 2010. 117(1): 243.

16. Djulbegovic, B., Hozo, I., Beckstead, J., Tsalatsanis, A. and Pauker, S., Dual processing model of medical decision-making. BMC Medical Informatics and Decision Making, 2012. 12(1): 94.

17. Sweeny, K., Melnyk, D., Miller, W. and Shepperd, J.A., Information avoidance: Who, what, when, and why. Review of General Psychology, 2010. 14(4): 340.

18. Howell, J.L. and J.A. Shepperd, Behavioral obligation and information avoidance. Annals of Behavioral Medicine, 2013: 1-6.

19. Case, D.O., Andrews, J.E., Johnson, J.D. and Allard, S.L., Avoiding versus seeking: the relationship of information seeking to avoidance, blunting, coping, dissonance, and related concepts. Journal of the Medical Library Association, 2005. 93(3): 353. 
20. Martin, S. and P.C. Smith, Rationing by waiting lists: an empirical investigation. Journal of Public Economics, 1999. 71(1): 141-164.

21. Yeung, R.Y., Leung, G.M., McGhee, S.M. and Johnston, J.M., Waiting time and doctor shopping in a mixed medical economy. Health Economics, 2004. 13(11): 1137-1144.

22. Gandhi, R., Razak, F., Davey, J.R., Rampersaud, Y.R. and Mahomed, N.N., Effect of sex and living arrangement on the timing and outcome of joint replacement surgery. Canadian Journal of Surgery, 2010. 53(1): 37.

23. Campbell, R., Evans, M., Tucker, M., Quilty, B., Dieppe, P. and Donovon, J.L., Why don't patients do their exercises? Understanding non-compliance with physiotherapy in patients with osteoarthritis of the knee. Journal of Epidemiology and Community Health, 2001. 55(2): 132-138.

24. Burke, M.A. and F. Heiland, The strength of social interactions and obesity among women, in Agent-based computational modelling. 2006, Springer. p. 117-137. 